\title{
Mapeamento de Gradiente Geotérmico do Estado do Rio Grande do Sul
}

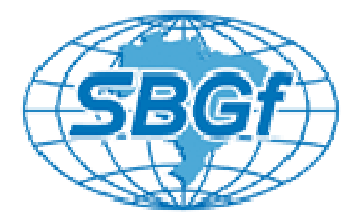

Jorge Luiz dos Santos Gomes (jorge.gomes@ufvim.edu.br), UFVJM; Antônio Jorge de Lima Gomes (antonio.gomes@ufvjm.edu.br), UFVJM; e Valiya Mannathal Hamza (hamza@on.br), Observatório Nacional/MCT.

Copyright 2014, SBGf - Sociedade Brasileira de Geofísica

Este texto foi preparado para a apresentação no VI Simpósio Brasileiro de Geofísica, Porto Alegre, 14 a 16 de outubro de 2014. Seu conteúdo foi revisado pelo Comitê Técnico do VI SimBGf, mas não necessariamente representa a opinião da SBGf ou de seus associados. É proibida a reprodução total ou parcial deste material para propósitos comerciais sem prévia autorização da SBGt.

\section{Resumo}

Apresentamos mapeamento regional do gradiente geotérmico do Estado do Rio Grande do Sul, cujos dados foram compilados com base em levantamentos de estudos geotérmicos realizados a partir da década de 1970. A compilação atual engloba medidas experimentais em 21 localidades, situadas em grande parte nas regiões Norte, Nordeste, Noroeste e Centro do Rio Grande do Sul. Constata-se que grande parte do estado apresentou gradientes geotérmicos na faixa de 24 a $32{ }^{\circ} \mathrm{C} / \mathrm{km}$, com valor médio de $28 \pm 4^{\circ} \mathrm{C} / \mathrm{km}$. Estes valores são típicos de áreas continentais com coberturas sedimentares, consideradas tectonicamente estáveis. Verifica-se que na parte central do Estado foram encontradas áreas com gradientes geotérmicos acima de $28^{\circ} \mathrm{C} / \mathrm{km}$, na região de interface do planalto basáltico com o afloramento da Bacia do Paraná. Este resultado observado na porção da Bacia do Paraná dentro do Estado, é considerado como resultado de refração de calor dos sedimentos da bacia, conjugado com o efeito de isolamento termal originado pelo baixo valor da condutividade térmica dos derrames de basalto da formação serra geral.

\section{Introdução}

O presente estudo tem como objetivo mapear 0 gradiente geotérmico do Estado do Rio Grande do Sul e interpretar o seu campo térmico regional, sobretudo na porção da Bacia do Paraná que está parcialmente situada dentro do Estado, e para isto foram utilizados resultados geotérmicos distribuídos dentro e fora do estado.

Este estudo faz parte de um projeto de pesquisa iniciado em 2004 pelo Laboratório de Geotermia do Observatório Nacional - ON/MCT e continuado pelo Laboratório da UFVJM no Campus do Mucuri em Teófilo Otoni, cujo objetivo é a quantificação de recursos geotermais da Bacia do Paraná e seu entorno, na qual está situado o Estado do Rio Grande do Sul.

A Bacia do Paraná está compreendida na parte meridional do Brasil com área de 1,1 milhões de $\mathrm{km}^{2}$, a metade oriental do Paraguai com $100 \mathrm{mil} \mathrm{km}^{2}$, parte da Argentina com 100 mil km² e Uruguai com 100 mil km², totalizando, aproximadamente, $1.400 .000 \mathrm{~km}^{2}$.

Os primeiros mapeamentos geotérmicos em escala regional de gradiente e fluxo geotérmico no Brasil, e também na América do Sul, foram efetuados por Hamza e Muñoz (1996), Hurter e Pollack (1996), Hamza et al (2005) e Gomes e Hamza (2004, 2005, 2006, 2007, 2008, 2009) e Gomes (2009).

\section{Metodologia}

Foram utilizados três métodos na determinação de gradientes geotérmicos e que foram respectivamente, o convencional (CVL), temperatura do fundo de poço de petróleo $(\mathrm{BHT})$ e o de temperatura estável do fundo de poço (CBT).

O método convencional (CVL) é utilizado para determinar gradientes em intervalos de profundidades selecionados, com base em resultados de perfilagem térmica, com correções dos efeitos de mudanças climáticas e de topografia de cada local.

O método de temperatura estável de fundo do poço (CBT) é utilizado nos locais em que o campo térmico do poço é alterado pelo fluxo de fluidos no seu interior.

O método de temperatura do fundo de poço de petróleo $(\mathrm{BHT})$ é utilizado em poços de petróleo para determinação de gradiente e fluxo geotérmico. Este apresenta incerteza no valor medido, originada principalmente pela utilização de termômetros com baixa exatidão para as medidas, que precisam ser corrigidas dos efeitos perturbadores das atividades de perfuração. Nos casos em que havia apenas uma medida de temperatura utilizou-se a relação empírica da AAPG (1976).

\section{Geologia e Geofísica regional}

De acordo com Kaul (1990), o estado do Rio Grande do Sul é constituído por terrenos rochosos cuja origem ou transformação retrocede aos mais diferentes períodos da história da crosta terrestre, trazendo o registro de distintos eventos geodinâmicos.

As rochas ígneas do período Pré-Cambriano fazem parte do Escudo Sul-Rio-Grandense e encontram-se muito erodidos. A altitude nessa região não ultrapassa os $600 \mathrm{~m}$.

Nas regiões Norte e Nordeste do Estado está situada parte da Bacia do Paraná, fazendo parte do Planalto Meridional, formada por rochas vulcânicas mesozóicas. Na porção NE do Estado encontram-se as partes altas do Estado com aproximadamente $1400 \mathrm{~m}$ de altitude.

A região central do Estado encontra-se a Cobertura de Sedimentos Cenozóicos, inseridos na Depressão Central, formando um corredor que corta toda a extensão E-W da área, através de terrenos de baixa altitude.

A região da Planície Costeira corresponde a uma faixa arenosa de $622 \mathrm{~km}$ de comprimento, com grande ocorrência de lagunas e lagoas, com sedimentos compreendidos dos períodos terciário e quaternário.

No que se refere à Gravimetria do Estado a anomalia bouguer apresenta uma forte correlação dos domínios gravimétricos de anomalia bouguer de uma forma geral, os domínios D1 e D2 estão associados ao 
vulcanismo básico (D1) e ácido (D2) da Formação Serra Geral (XAVIER, 2009).

Na figura (1) apresenta-se mapa Geológico modificado de CPRM (2014), onde as cores verdes apresentam o Planalto Basáltico, os tons avermelhados 0 Embasamento Cristalino e os tons amarelos a planície costeira.

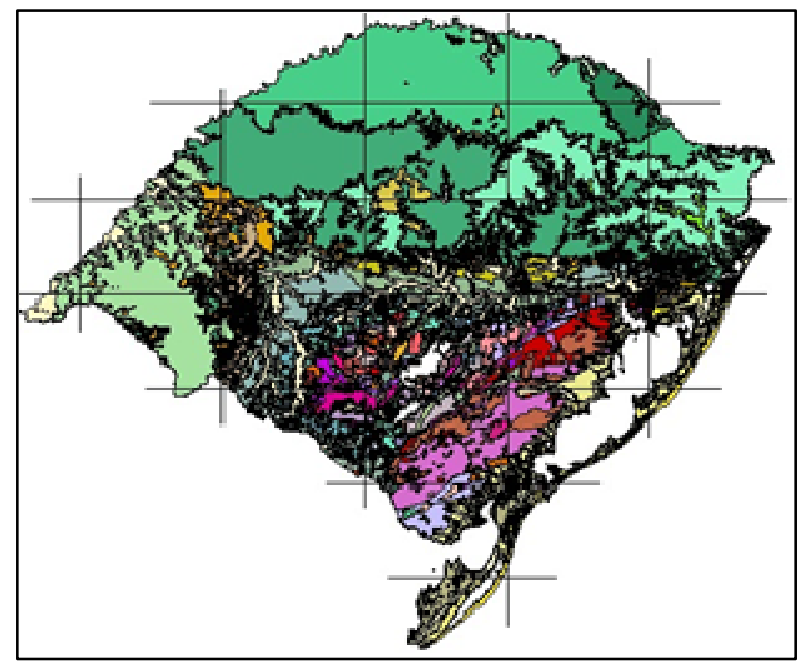

Figura 1 - Mapa da geologia simplificada do Estado do Rio Grande do Sul (modificado de CPRM, 2014).

\section{Resultados}

Apresenta-se na Figura (2) a localização dos 21 dados geotérmicos compilados dentro do Estado do Rio Grande do Sul. Destes, 12 resultados são do método BHT, 8 dados são do CVL e 1 CBT.

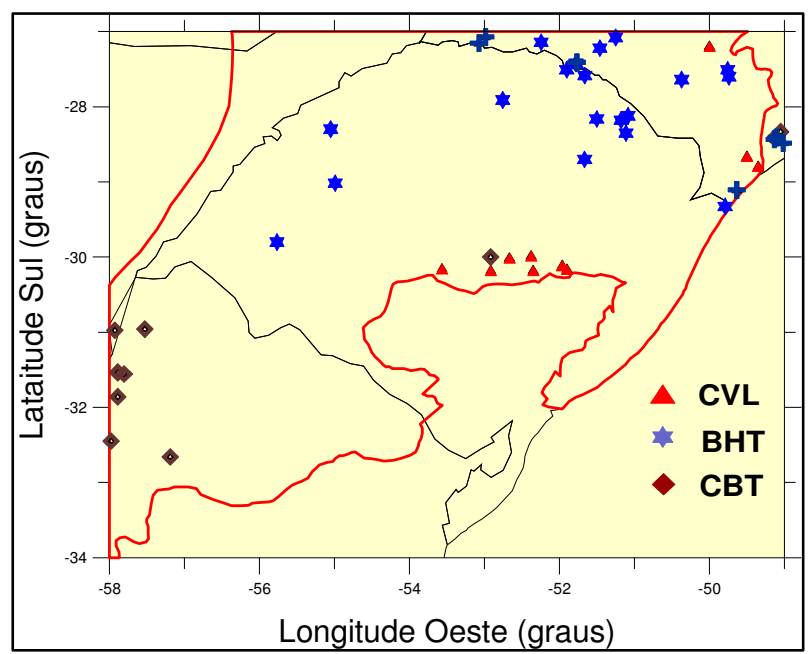

Figura 2 - Localização dos dados geotérmicos por tipo de gradiente, no Estado do Rio Grande do Sul e seu entorno, com destaque em vermelho para o contorno da porção da Bacia do Paraná.

Para um melhor entendimento do campo termal do Estado e suas inferências regionais, ainda, nesta compilação, apresentam-se os dados estudados em 21 locais no Estado e também mais 37 dados em áreas vizinhas do Uruguai, Paraguai e Argentina, totalizando 58 localidades com base em Gomes e Hamza (2008).

Apresentam-se na Tabela (1) todos os gradientes geotérmicos obtidos dentro do Estado. Os valores dos demais gradientes do entorno constam da publicação de Gomes e Hamza (2008) e Gomes (2009).

Tabela 1 - Gradientes Geotérmicos por local e seus respectivos desvio padrão (Г).

\begin{tabular}{|c|c|c|c|c|}
\hline \multirow{2}{*}{ Município } & \multicolumn{2}{|c|}{ Coordenadas } & \multicolumn{2}{|c|}{$\Gamma\left({ }^{\circ} \mathrm{C} / \mathrm{km}\right)$} \\
\hline & Long. & Lat. & Médio & s \\
\hline Alegrete & $-55,77$ & $-29,80$ & 18,1 & 0,9 \\
\hline Butiá & $-51,96$ & $-30,12$ & 28,8 & 0,9 \\
\hline Butiá & $-51,90$ & $-30,17$ & 38,8 & 0,7 \\
\hline Butiá & $-51,96$ & $-30,12$ & 33,4 & 0,9 \\
\hline $\begin{array}{c}\text { Cachoeira do } \\
\text { Sul }\end{array}$ & $-52,92$ & $-30,00$ & 20,3 & 0,6 \\
\hline Esmeralda & $-51,18$ & $-28,18$ & 23,0 & 0,7 \\
\hline Esmeralda & $-51,09$ & $-28,12$ & 23,0 & 0,9 \\
\hline Itacurubi & $-54,99$ & $-29,02$ & 19,9 & 0,6 \\
\hline $\begin{array}{c}\text { Lagoa } \\
\text { Vermelha }\end{array}$ & $-51,50$ & $-28,16$ & 20,2 & 0,9 \\
\hline Machadinho & $-51,66$ & $-27,59$ & 27,9 & 0,9 \\
\hline M. Ramos & $-51,90$ & $-27,51$ & 28,6 & 0,9 \\
\hline Muitos Capões & $-51,11$ & $-28,35$ & 27,8 & 0,8 \\
\hline Nova Bassano & $-51,66$ & $-28,70$ & 25,7 & 0,8 \\
\hline Paraíso do Sul & $-52,92$ & $-30,18$ & 22,9 & 0,7 \\
\hline Rio Pardo & $-52,38$ & $-29,99$ & 41,0 & 1,2 \\
\hline Rio Pardo & $-52,67$ & $-30,02$ & 40,3 & 1,2 \\
\hline Rio Pardo & $-52,35$ & $-30,18$ & 40,3 & 0,8 \\
\hline Ronda Alta & $-52,76$ & $-27,91$ & 20,6 & 0,9 \\
\hline $\begin{array}{c}\text { São L. } \\
\text { Gonzaga }\end{array}$ & $-55,05$ & $-28,30$ & 19,4 & 0,6 \\
\hline São Sepé & $-53,57$ & $-30,16$ & 36,0 & 1,4 \\
\hline Torres & $-49,79$ & $-29,33$ & 31,7 & 1 \\
\hline
\end{tabular}

O mapeamento dos resultados é apresentado nas Figuras (3) e (4). Na primeira figura foram considerados apenas os dados situados dentro do Estado e na segunda figura foram também considerados os dados dos estados e países vizinhos.

Os resultados obtidos para o gradiente geotérmico dentro do Estado do Rio Grande do Sul apresentaram valores situados no intervalo entre $18,1^{\circ} \mathrm{O} / \mathrm{km}$ em Alegrete e máximo de $41^{\circ} \mathrm{C} / \mathrm{km}$ em Rio Pardo.

Os resultados do mapeamento indicam que o os menores valores encontrados do gradiente geotérmico estão situados na parte interna da Bacia do Paraná na 
região compreendida pela formação Serra Geral, o que também é observado nas áreas vizinhas, conforme é constatado no Estado de Santa Catarina e no país vizinho Uruguai, neste sentido a porção da Bacia do Paraná possivelmente é afetada por percolação de fluídos em seu interior.

Segundo Almeida (1980) e Zalán et al. (1988 e 1990), a Bacia do Paraná é constituída por um pacote de rochas sedimentares e vulcânicas.

Possivelmente a presença dos basaltos com baixa condutividade térmica, cujo valor médio é de 1,81 $\mathrm{W} / \mathrm{m} . \mathrm{K}$, faz com que esta camada se comporte como um isolante térmico, perturbando 0 valor da temperatura nas camadas mais rasas da Bacia, resultando em maiores valores de gradiente no método CVL em comparação com o tipo BHT (GOMES, 2009).

Os resultados do gradiente do tipo CVL estão compreendidos entre $28,8^{\circ} \mathrm{C} / \mathrm{km}$ em Butiá e $41^{\circ} \mathrm{C} / \mathrm{km}$ em Rio Pardo.

Os valores encontrados para o tipo $\mathrm{BHT}$, ficaram compreendidos entre $18,1^{\circ} \mathrm{\circ} / \mathrm{km}$ em Alegrete e 31,7 ${ }^{\circ} \mathrm{C} / \mathrm{km}$ em Torres.

Constatamos que o comportamento térmico observado nas regiões da borda da Bacia do Paraná são de baixa entalpia, cujas águas quentes apresentam temperaturas na superfície de até $45^{\circ} \mathrm{C}$, resultando em gradientes médios de $28^{\circ} \mathrm{C}$.

\section{Discussão e Conclusões}

Os mapas das figuras (3) e (4) indicam que as regiões próximas as regiões próximas ao centro do Estado do Rio Grande do, situadas, compreendidas no afloramento gonduânico da Bacia do Paraná, possuem valores mais altos de gradiente que as demais regiões da parte interna da Bacia. Esses valores seguem altos no sentido da plataforma continental, cujos valores foram, respectivamente, maiores que $28^{\circ} \mathrm{C} / \mathrm{km}$ e que alcançam até $41^{\circ} \mathrm{C} / \mathrm{km}$.

As regiões internas próximas à borda da Bacia apresentam valores intermediários de gradiente compreendidos entre 26 e $32{ }^{\circ} \mathrm{C} / \mathrm{km}$.

A borda da Bacia apresenta os mais altos valores de gradiente e fluxo térmico respectivamente compreendidos entre 28 e $42^{\circ} \mathrm{C} / \mathrm{km}$.

Os poços mais profundos com os resultados $\mathrm{BHT}$ apontam valores menores de gradiente geotérmico do que os mais rasos, possivelmente devido a uma menor perturbação dos fluídos presentes nos arenitos e folhelhos da porção sedimentar mais profunda da bacia.

Estudos avançados com mais detalhes poderão esclarecer melhor a diferença de valores entre a parte mais rasa e mais profunda da Bacia no Rio Grande do Sul.

Os valores do gradiente geotérmico observados na faixa gonduânica apontam que estes locais apresentam possibilidade de aproveitamento geotermal, tanto para atividades balneárias quanto para uso direto.

\section{Referências}

American Association of Petroleum Geologists, 1976. Basic Data from AAPG Geothermal Survey of North America, University of Oklahoma, Norman, Okla, USA.

Del Rey, A.C. and Hamza, V.M., 1989. Terrestrial Heat Flow variations in the northeastern parts of the state of São Paulo : A case for transport of geothermal heat by interfracture fluid flows, In Hydrogeological Regimes and their subsurface thermal effects, Geophysical Monograph, 47, American Geophysical Union, p. 137-148.

Eston, S.M., Hamza, V.M. e Becker, E.A., 1981, Pesquisas Geotérmicas na Exploração de Hidrocarbonetos na Bacia do Paraná, Relatório 16.379, Instituto de Pesquisas Tecnológicas do Estado de São Paulo-IPT.

Eston, S.M., Hamza, V.M. e Becker, E.A., 1981, Pesquisas Geotérmicas na Exploração de Hidrocarbonetos na Bacia do Paraná, Relatório 16.379, Instituto de Pesquisas Tecnológicas do Estado de São Paulo-IPT.

Gomes, A.J.L. e Hamza, V.M., 2004. Mapeamento de Gradientes Geotérmicos no Estado de São Paulo, In: 1 Simpósio Regional de Geofísica, Anais, Cd-rom, São Paulo.

Gomes, A.J.L. and Hamza, V.M., 2005. Gradiente e Fluxo Geotérmico do Estado de Santa Catarina, 9o International Congress of the Brazilian Geophysical, Salvador, Brasil.

Gomes, A.J.L. and Hamza, V.M., 2006. Gradiente e Fluxo Geotérmico no Estado do Paraná. In: II Simpósio de Geofísica da SBGf, 2006, Natal.

Gomes, A.J.L. and Hamza, V.M., 2007. Gradiente e Fluxo Geotérmico nas regiões Norte e Noroeste da Bacia do Paraná. In: 10 ICSBGf,Rio de Janeiro.

Gomes, A.J.L. and Hamza, V.M., 2008. Distribuição Regional de Gradiente e Fluxo Geotérmico no Estado do Rio Grande do Sul incluindo também áreas vizinhas do Uruguai e da Argentina. In: III Simpósio de Geofísica da SBGf, 2008, Belém.

Gomes, Gomes, A.J.L. Avaliação de Recursos Geotermais da Bacia do Paraná, Tese de Doutorado, Observatório Nacional, 2009.

Hamza, V.M., Silva Dias, F.J.S., Gomes, A.J.L. and Terceros, Z.G.D., 2005. Numerical and Functional Representations of Regional Heat Flow in South America, Physics of the Earth and Planetary Interiors, Volume 152, 4, p.223-256.

Hamza, V.M. and Muñoz, M., 1996. Heat Flow map of

South America, Geothermics, Vol. 25, no 6, pp. 599646.

Hurter, S.J. and Pollack, H.N., 1996. Terrestrial heat flow in the Paraná basin, Southern Brazil, J. Geophysics.Res., 101, p.8659-8672.

Xavier, M. B., 2009, Detecção de Erros Grosseiros em Banco de Dados Gravimétrico Terrestre do Estado Do Rio Grande Do Sul, Tese de Mestrado, UFRGS. 


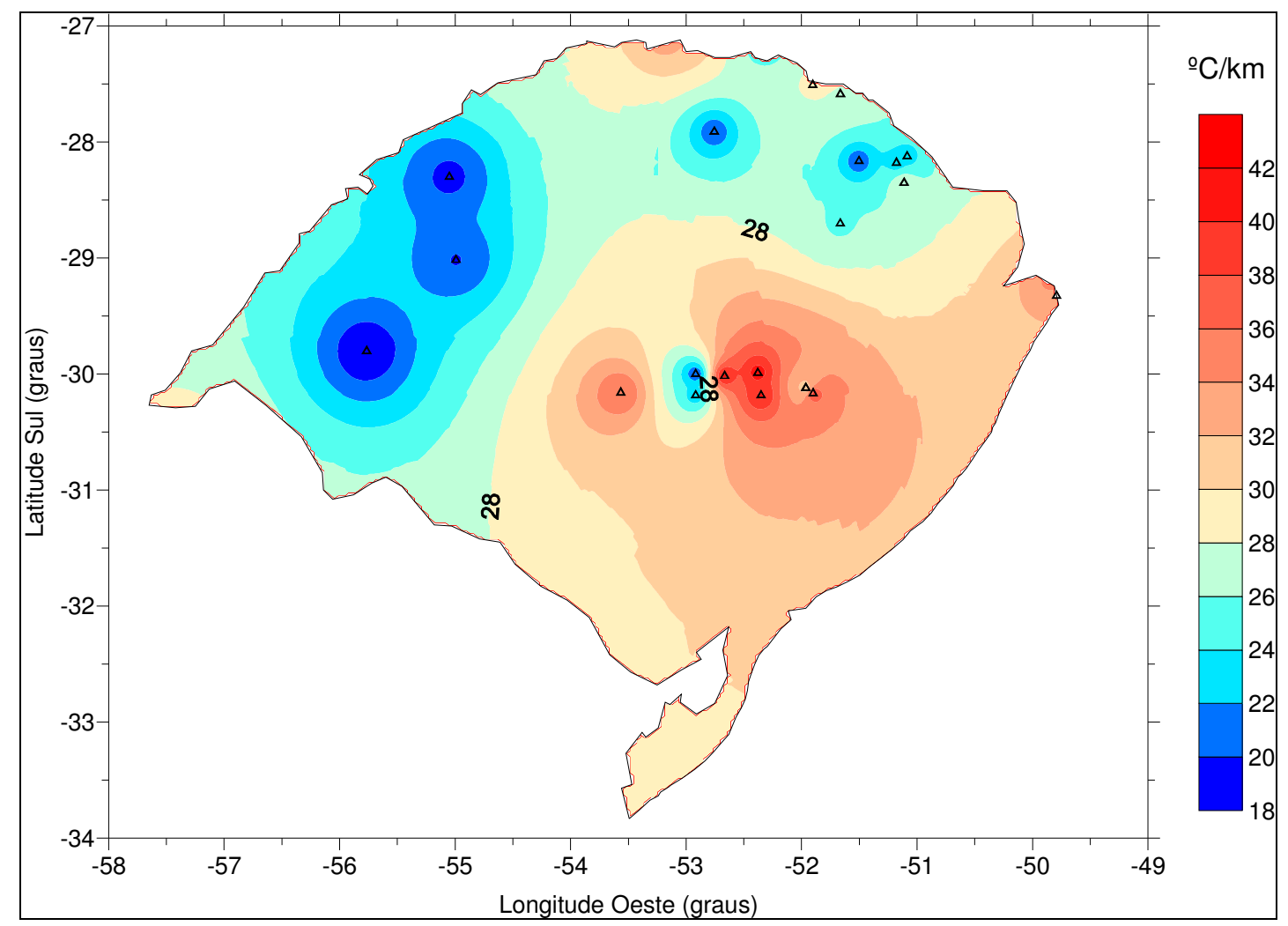

Figura 3 - Mapeamento regional de gradiente geotérmico considerando apenas os dados situados dentro do Estado.

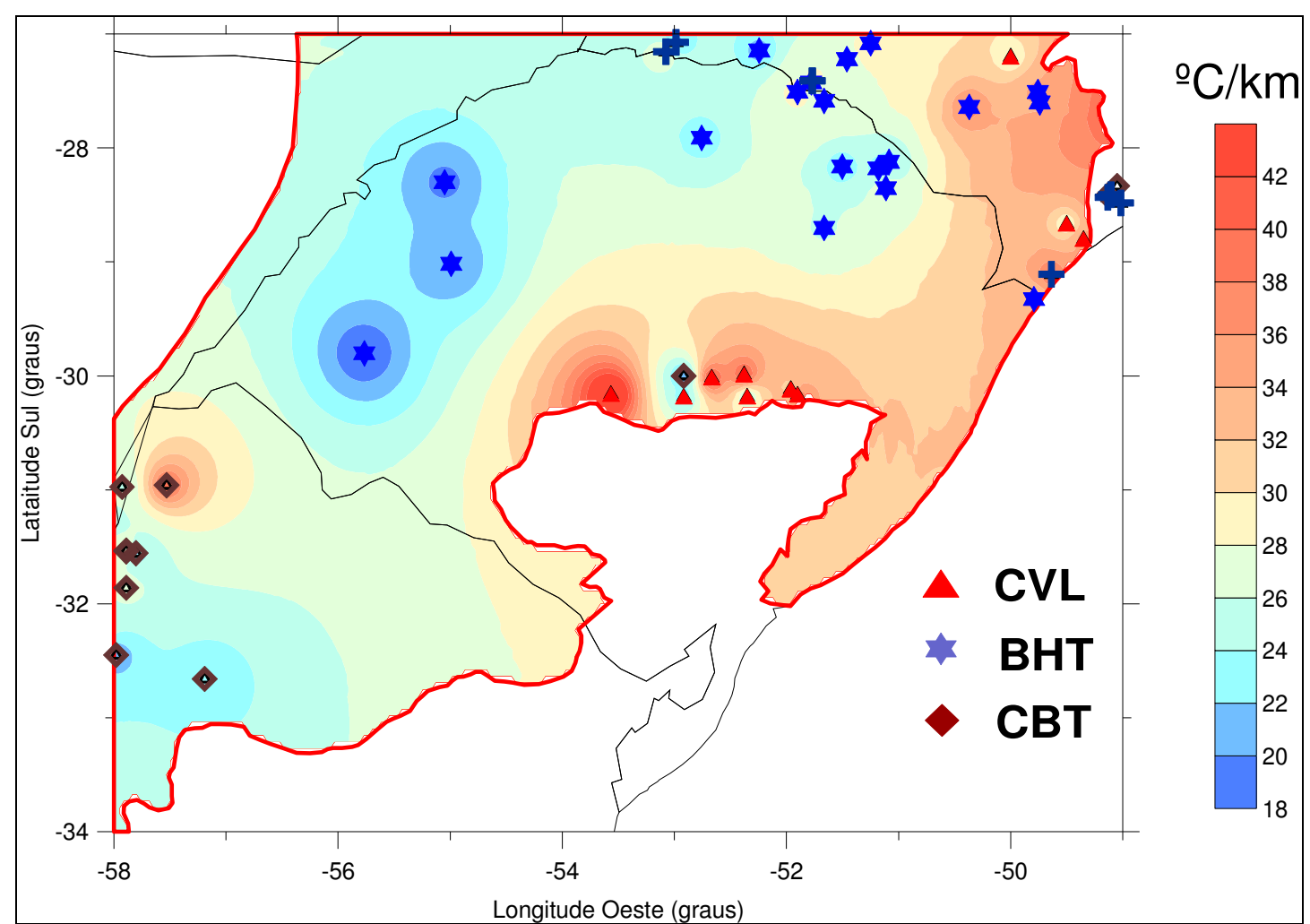

Figura 4 - Mapeamento regional de gradiente geotérmico limitado ao contorno da Bacia do Paraná dentro do Rio Grande do Sul considerando os dados CVL, BHT e CBT, situados dentro do Estado e no seu entorno. 